\title{
Functional Signal- and Paradigm-Dependent Linear Relationships between Synaptic Activity and Hemodynamic Responses in Rat Somatosensory Cortex
}

\author{
Masahito Nemoto, Sameer Sheth, Michael Guiou, Nader Pouratian, James W. Y. Chen, and Arthur W. Toga \\ Laboratory of Neuroimaging, Department of Neurology, University of California, Los Angeles, School of Medicine, Los Angeles, California 90024
}

\begin{abstract}
Linear relationships between synaptic activity and hemodynamic responses are critically dependent on functional signal etiology and paradigm. To investigate these relationships, we simultaneously measured local field potentials (FPs) and optical intrinsic signals in rat somatosensory cortex while delivering a small number of electrical pulses to the hindpaw with varied stimulus intensity, number, and interstimulus interval. We used 570 and $610 \mathrm{~nm}$ optical signals to estimate cerebral blood volume (CBV) and oxygenation, respectively.

The spatiotemporal evolution patterns and trial-by-trial correlation analyses revealed that CBV-related optical signals have higher fidelity to summed evoked FPs ( $\Sigma$ FPs) than oxygenation-derived signals. CBV-related signals even correlated with minute $\Sigma$ FP fluctuations within trials of the same stimulus condition. Furthermore, hemodynamic signals (CBV and late oxygenation signals) increased linearly with $\Sigma F P$ while varying stimulus number, but they exhibited a threshold and steeper gradient while varying stimulus intensity, suggesting insufficiency of the homogeneity property of linear systems and the importance of spatiotemporal coherence of neuronal population activity in hemodynamic response formation. These stimulus paradigm-dependent linear and nonlinear relationships demonstrate that simple subtraction-based analyses of hemodynamic signals produced by complex stimulus paradigms may not reflect a difference in $\Sigma F P$ s between paradigms. Functional signal- and paradigm-dependent linearity have potentially profound implications for the interpretation of perfusion-based functional signals.
\end{abstract}

Key words: optical imaging; somatosensory; blood volume; neurovascular coupling; linear systems; fMRI

\section{Introduction}

Recently, several studies have investigated the relationship between neuronal activity and hemodynamic responses using simultaneous recordings of electrophysiological signals and functional magnetic resonance imaging (fMRI) blood oxygenation level-dependent (BOLD) signals (Ogawa et al., 2000; Logothetis et al., 2001), laser-Doppler cerebral blood flow (CBF) signals (Mathiesen et al., 1998; Ngai et al., 1999; Nielsen and Lauritzen, 2001), and optical intrinsic signals (Sheth et al., 2003). The results showed that hemodynamic responses correlate significantly with integrated synaptic activity. They also demonstrated the limitations inherent in applying these signals to basic neuroscience, because hemodynamic responses reflect only limited aspects of cellular activities in cognitive processes. Hemodynamically derived signals are complicated, because they spread in time and space compared with neuronal activity, resulting in temporally and spatially overlapping responses. To deconvolute the signals, more information is required concerning their principles of superposition in time and space, which may differ depending on

Received 0ct. 30, 2003; revised Feb. 6, 2004; accepted March 8, 2004.

This work was supported by National Institutes of Health Grants MH/NS52083, MH67432, and GM08042. We thank P. Thompson and F. Miwakeichi for advice on statistical analysis.

Correspondence should be addressed to Dr. Arthur W. Toga, Laboratory of Neuroimaging, 710 Westwood Plaza, Suite 4-238, Mail Code 176919, Los Angeles, CA 90024-1769. E-mail: toga@loni.ucla.edu.

D01:10.1523/JNEUROSCI.4870-03.2004

Copyright $\odot 2004$ Society for Neuroscience $\quad$ 0270-6474/04/243850-12\$15.00/0 signal etiology, vascular compartments, physiological conditions, and functional paradigms. Because of the complexity of this task, functional signals demonstrating high contrast-to-noise ratios (CNRs) and simple principles of spatial and temporal superposition are preferable. Deoxyhemoglobin-weighted signals, such as fMRI BOLD and optical intrinsic signals in the $600 \mathrm{~nm}$ range, have proven to be valuable mapping signals over the past decade. However, some studies have identified nonlinear components (Rees et al., 1997; Miller et al., 2001), spatial coupling and uncoupling (Disbrow et al., 2000; Duong et al., 2000, 2001; Kim et al., 2000), and CNRs lower than those of cerebral blood volume (CBV)-weighted response signals (Mandeville et al., 1998; Kim and Ugurbil, 2003; Sheth et al., 2003).

Here, we focused on how metabolic and hemodynamic signals evolve in time and space and how linearly they correlate with underlying neuronal activity. To investigate their fundamental relationships on a microscopic scale, we used a small number of electrical pulses to the rat hindpaw and simultaneously recorded local field potentials (FPs) and optical intrinsic signals in the somatosensory cortex. Neuronal activity is a complex phenomenon that can be observed via electrophysiological signals such as extracellular FPs, single- and multiple-unit activity, and membrane potentials. These measurable parameters reflect different aspects of excitatory and inhibitory synaptic activity or subthreshold and spiking activity at the level from an individual cell to neural ensemble on the basis of functional structures (Logoth- 
etis, 2002). Here, we chose summed evoked FPs (¿FPs) as an indicator of integrated synaptic activity (Mathiesen et al., 1998) and evaluated the correlations between the $\Sigma$ FPs and optical signal responses. We also determined whether their relationship showed evidence of properties of a linear system: homogeneity and additivity properties. These properties were examined with three stimulus paradigms: variable stimulus intensity, number, and interstimulus interval (ISI). We used $570 \mathrm{~nm}$ (an isosbestic point of oxyhemoglobin and deoxyhemoglobin) optical signals to estimate changes in total hemoglobin concentration associated with $\mathrm{CBV}$ and used $610 \mathrm{~nm}$ (deoxyhemoglobin-dominant) signals to estimate the balance of oxygen metabolism, CBF and $\mathrm{CBV}$. The spatiotemporal evolution of these signals was interpreted with regard to fMRI BOLD and CBV- and CBF-related signals.

\section{Materials and Methods}

\section{Animal preparation}

Nine adult male Sprague Dawley rats (330-500 gm; Charles River Laboratories, Wilmington, MA) were initially anesthetized with halothane (4-5\% during induction; $1.5 \%$ during surgery) and maintained with $0.75-1.5 \%$ enflurane in $40 \% \mathrm{O}_{2}$ and $60 \% \mathrm{~N}_{2}$ during data collection. Rats breathed spontaneously, and rectal temperature was maintained at $37^{\circ} \mathrm{C}$ with a homeothermic blanket (Harvard Bioscience, Holliston, MA). The inspired enflurane concentration was regulated to keep a relatively constant anesthetic depth (Guedel stage III-2) assessed by respiration rate and moderate foot pinch withdrawal (Friedberg et al., 1999). Rats were placed in a stereotaxic frame (David Kopf Instruments, Tujunga, CA), and lidocaine was used at the operation sites for local anesthesia. The skull was exposed, and the right parietal bone was thinned $(5 \times 7 \mathrm{~mm})$ over the sensorimotor cortex using a dental drill under continuous cooling with physiologic saline. Experimental protocols were approved by the Animal Research Committee of the University of California, Los Angeles.

\section{Stimulation procedures and electrophysiological recordings}

Electrical pulses ( $1 \mathrm{msec}$ each) were delivered via a pair of needle electrodes inserted under the skin of the plantar and ankle region in the contralateral hindpaw. To modulate total neuronal activities per unit time, we varied the parameters of stimulus intensity, number, and ISI independently.

Protocol 1 (variable stimulus intensity). We varied stimulus amplitude $(0.4,0.8$, and $1.6 \mathrm{~mA})$ while keeping number (five pulses) and ISI (200 $\mathrm{msec}$ ) constant. In protocol 1, individual neuronal responses were modulated at the same rate with varying stimulus intensity (see Results).

Protocol 2 (variable stimulus number). We varied pulse number (one, three, and six) while keeping intensity $(0.8 \mathrm{~mA})$ and ISI $(200 \mathrm{msec})$ constant. In protocol 2 , total neuronal activity per unit time is modulated by varying stimulus number rather than individual neuronal responses.

Protocol 3 (variable ISI). We varied ISI (200, 150, 100, and $66 \mathrm{msec}$ ) while keeping number (two pulses) and intensity $(0.8 \mathrm{~mA})$ constant. The second neuronal response is strongly suppressed by thalamocortical and/or intracortical inhibitory circuits (Simons and Carvell, 1989; Moore et al., 1999; Castro-Alamancos and Oldford, 2002; Nicolelis and Fanselow, 2002) with decreasing ISI.

Stimuli were presented every $30 \mathrm{sec}$ in the above order or pseudorandomly using three stimulators (ISO-flex; A.M.P.I., Jerusalem, Israel) triggered by a pulse generator (Master 8; A.M.P.I.). Extracellular FPs, obtained simultaneously with optical imaging, were recorded using a tungsten unipolar microelectrode $\left(0.2-0.4 \mathrm{M} \Omega\right.$ at $1 \mathrm{kHz}, 12^{\circ}$ tapered tip, $250 \mu \mathrm{m}$ diameter; A-M Systems, Carlsborg, WA). Before inserting the microelectrode, we acquired single trial images of optical responses to a $0.8 \mathrm{~mA}$, five pulse, $200 \mathrm{msec}$ ISI stimulus. We drilled a small burr hole near the center of the optical response, avoiding cortical vessels. The microelectrode was advanced to $400-600 \mu \mathrm{m}$ through the burr hole. An $\mathrm{Ag}-\mathrm{AgCl}$ reference electrode was placed in the occipital muscle. FP signals were amplified 1000 times (model SCXI-1120; National Instruments, Austin, TX), low-pass-filtered at $10 \mathrm{kHz}$, and digitally sampled at
$1 \mathrm{kHz}$ using a personal computer running LabView (version 5.1; National Instruments).

\section{Optical imaging}

Rats were placed on the stage of a stereoscopic zoom microscope (model SMZ1500; Nikon, Melville, NY) equipped with a cooled 16-bit CCD camera (TE/CCD-576EFT; Princeton Instruments, Trenton, NJ). The cortex was epi-illuminated with filtered light from a voltage-regulated quartz-tungsten-halogen light source (model PL900; Dolan-Jenner, Lawrence, MA). We used transmission filters centered at $570 \mathrm{~nm}$ [full width at half maximum (FWHM), $10 \mathrm{~nm}$ ] and $610 \mathrm{~nm}$ (FWHM, $10 \mathrm{~nm}$ ). Filters were alternated every trial by a filter changer (Lambda 10-2; Sutter Instruments, Novato, CA) to reduce the time lag between image acquisition at the two wavelengths so that changes in physiological conditions had a similar effect on both signals. Eighty images ( 40 prestimulus and 40 poststimulus) were acquired every $250 \mathrm{msec}$ for $20 \mathrm{sec}$ with an exposure time of $100 \mathrm{msec}$. Image dimensions were 192 (width) $\times 144$ (height) covering a $9.6 \times 7.2 \mathrm{~mm}^{2}$ field. The intertrial interval was $30 \mathrm{sec}$. A minimum of 15 trials were collected for each stimulus condition and wavelength.

\section{Data analysis}

The magnitude of optical intrinsic signals was calculated as fractional change in reflected light intensity relative to prestimulus baseline. We analyzed images by pixel-by-pixel division and subtraction ( $\Delta$ ratio image) to detect the intrinsic signals as follows:

$$
\begin{aligned}
& \operatorname{ratio}_{\lambda}(t)=I_{\lambda}(t) / I_{\lambda}^{\text {base }} \\
& \Delta \operatorname{ratio}_{\lambda}(t)=\operatorname{ratio}_{\lambda}(t)-\operatorname{ratio}_{\lambda}(-0.25) \\
& I_{\lambda}^{\text {base }}=\left\{I_{\lambda}(-1.0)+I_{\lambda}(-0.75)+\right. \\
&\left.I_{\lambda}(-0.5)+I_{\lambda}(-0.25)\right\} / 4
\end{aligned}
$$

$$
\lambda=570,610 \mathrm{~nm},
$$

where $I_{\lambda}(t)$ represents reflected light intensity in the image $t$ seconds after stimulus onset at wavelength $\lambda$, and $I_{\lambda}{ }^{\text {base }}$ is the prestimulus baseline intensity determined by averaging four images collected $\leq 1 \mathrm{sec}$ before the stimulus onset. Generating ratio images corrects for uneven illumination and provides a time course closely related to changes in absorber concentration. Using a baseline averaged over a $1 \mathrm{sec}$ prestimulus period $\left(I_{\lambda}{ }^{\text {base }}\right)$ diminishes high-frequency spatial noise (shot noise), and subtracting the ratio ${ }_{\lambda}(-0.25)$ image provides zero-point correction to detect stimulus-specific signals. Regions of interest (ROIs) were determined from the response to a $0.8 \mathrm{~mA}, 200 \mathrm{msec}$ ISI, five-pulse stimulus that we defined (for subsequent normalization procedures) as the standard stimulation. An automated program (MatLab; MathWorks, Natick, MA) determined the 0.5-, 1-, 1.5-, and 2-mm-diameter circular ROI position resulting in maximum response values in the average $\Delta$ ratio images collected $0.5-1.5 \mathrm{sec}$ after stimulus onset (Chen-Bee et al., 2000). To investigate the time dependence of maximum response locations, we generated ROIs during one later time point for each wavelength: $2-3 \mathrm{sec}$ (peak epoch) for $570 \mathrm{~nm}$ responses and 3.5-4.5 sec (late positive peak epoch) for $610 \mathrm{~nm}$ responses. We generated time courses for all of the stimulation protocols by applying these ROIs to the image sequence. In general, the time courses of these optical responses are more strongly affected by the changes in the response areal extent with increasing ROI size. We also calculated the temporal profiles of horizontal and vertical cross sections of the response (width, six pixels) passing through the center of the $0.5 \mathrm{~mm}$ ROI to investigate spatiotemporal dynamics of optical intrinsic signals. Response areal extent was quantified with both absolute thresholds (Chen-Bee et al., 2000) and statistical significance thresholds. In both cases, we considered three ratio images: the image collected $0.25 \mathrm{sec}$ before stimulus onset $\left[\operatorname{ratio}_{\lambda}(-0.25)\right]$, the peak image $\left[\operatorname{ratio}_{\lambda}\left(t_{\mathrm{p}}\right)\right]$, and the image collected $0.25 \mathrm{sec}$ before the peak $\left[\operatorname{ratio}_{\lambda}\left(t_{\mathrm{p}}-0.25\right)\right]$. Images were first processed with a three-pixel FWHM Gaussian filter to reduce high-frequency spatial noise. Absolute thresholds were set at various levels above baseline $\left[\operatorname{ratio}_{\lambda}(-0.25)\right]$ and applied to the average of $\Delta \operatorname{ratio}_{\lambda}\left(t_{\mathrm{p}}\right)$ and $\Delta \operatorname{ratio}\left(t_{\mathrm{p}}-0.25\right)$. For statistical 
mapping, we performed a pixel-by-pixel ANOVA test on these three ratio images in a trial set. Pixels were thus assigned a high degree of significance if their variance within a trial was high compared with their variance between trials. This process attributed high significance to pixels that responded strongly to the stimulation and low significance to those that showed large trial-to-trial variability. With this method we were able to determine statistical significance ( $p$ value) maps. Both areal quantification procedures were applied to the $570 \mathrm{~nm}$ responses and both phases of the biphasic $610 \mathrm{~nm}$ responses. Because of the lack of a universal threshold level, we calculated areal extent using several threshold levels of absolute $\Delta$ ratio values and statistical $p$ values and then used three representative thresholds for correlation analyses.

Evoked FPs consisted of an initial large negative deflection followed by a smaller positive waveform. We first measured the amplitude of each evoked potential from baseline to the negative peak without averaging and then calculated the summation of the evoked $\Sigma F P$ in each trial. Extracellular FPs represent a weighted average of synchronized synaptic inputs of a neural population within several millimeters of the electrode tip (Logothetis, 2002). Their summation per unit time is a good indicator of integrated synaptic activity and its accumulated influence on CBF and metabolism (Mathiesen et al., 1998; Ngai et al., 1999; Nielsen and Lauritzen, 2001).

Correlation between $\Sigma F P$ and optical signal responses $\left[\Delta \operatorname{ratio}_{\lambda}\left(t_{\mathrm{p}}\right)\right.$ within ROIs and areal extents] was evaluated by a least-squares regression analysis based on individual trial data and trial-averaged data. Raw and normalized data were analyzed because the response size varied across individual animals. A single electrical pulse (or whisker deflection) generates small changes in reflected light intensity originating from hemodynamic and metabolic processes (Polley et al., 1999; Martindale et al., 2003), which we defined as the unit response of optical intrinsic signals. We normalized each response using average responses of $\Sigma F P$ s and optical signals evoked by the standard stimulation $(0.8$ $\mathrm{mA}, 200 \mathrm{msec}$ ISI, and five pulses) in each animal. We defined the magnitudes of these standard responses as five arbitrary units and then rescaled all data. Assuming that the slow metabolic and hemodynamic unit responses to single-pulse stimuli temporally and spatially overlap during the brief repetitive stimulation, we simply compared $\Sigma F P$ with the magnitude and areal extent of peak optical signal responses without temporal integration (Heeger and Ress, 2002).

We then examined whether the neuronal-hemodynamic relationship showed properties of a linear system. A system is a mathematical model represented as an operator $S$ in which an input signal $f(x)$ is transformed into an output signal $g(x)$ as follows: $S[f(x)]=g(x)$. Given $S\left[f_{1}(x)\right]=$ $g_{1}(x)$ and $S\left[f_{2}(x)\right]=g_{2}(x)$, the system is linear if its operator $S$ satisfies the following two conditions: (1) homogeneity (scaling), $S\left[\alpha_{1} f_{1}(x)\right]=\alpha_{1} S$ $\left[f_{1}(x)\right]=\alpha_{1} g_{1}(x)$ for any signals $f_{1}(x)$ and scalar $\alpha_{1}$; and (2) additivity, $S$ $\left[f_{1}(x)+f_{2}(x)\right]=S\left[f_{1}(x)\right]+S\left[f_{2}(x)\right]=g_{1}(x)+g_{2}(x)$ for any input $f_{1}(x)$ and $f_{2}(x)$. These two conditions can be combined into a single condition (superposition) as $S\left[\alpha_{1} f_{1}(x)+\alpha_{2} f_{2}(x)\right]=\alpha_{1} S\left[f_{1}(x)\right]+\alpha_{2} S\left[f_{2}(x)\right]=$ $\alpha_{1} g_{1}(x)+\alpha_{2} g_{2}(x)$, where $\alpha_{1}$ and $\alpha_{2}$ are arbitrary scalars. In the present study, $f(x)$ and $g(x)$ represent FPs and optical signals at a given location or bottom; see Discussion).
$570 \mathrm{~nm}$

$0.5-1.5 \mathrm{~s}$

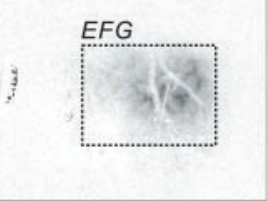

$2.0-3.0 \mathrm{~s}$

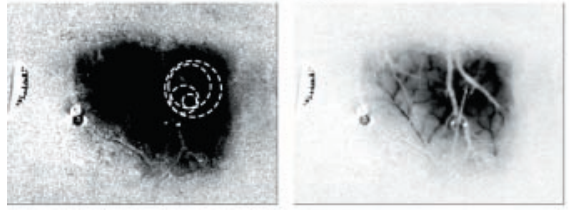

$7.0-8.0 s$
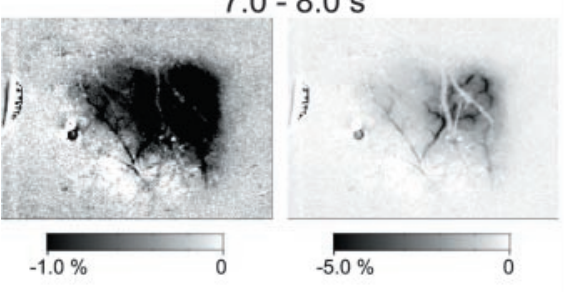

B| C

$610 \mathrm{~nm}$

$-1.5 \mathrm{~s}$

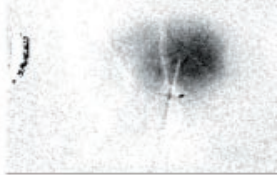

$1.25-2.25 \mathrm{~s}$
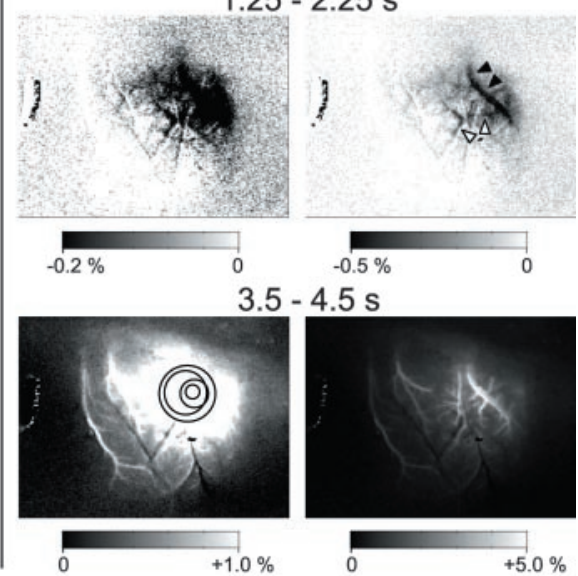

$3.5-4.5 \mathrm{~s}$

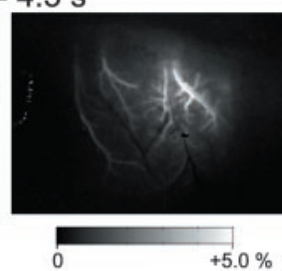

G
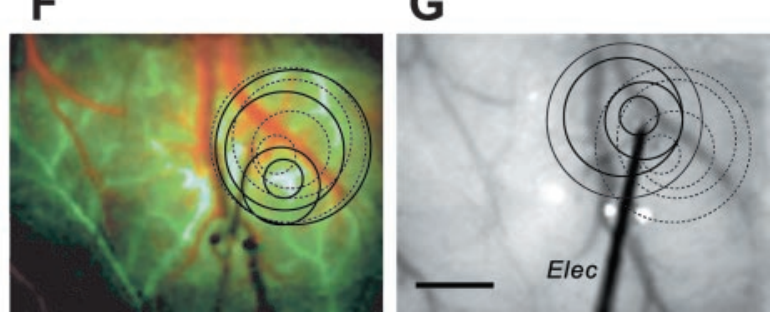

Figure 1. ROI determination and spatiotemporal dynamics of 570 and $610 \mathrm{~nm}$ optical intrinsic signals. ROls were determined from a response to a $0.8 \mathrm{~mA}$, five pulse, $200 \mathrm{msec}$ ISI stimulus (the standard stimulation). $A-D, 0$ ptical responses are displayed at $(B, D)$. The 0.5-, 1-, 1.5-, and 2-mm-diameter circular ROls $(A, C)$ were determined to record the maximum response values in lines at the top of $B . F, A$ composite image made from the middle of $B$ (green channel) and the bottom of $D$ (red channel) asizing activated arterioles (green) and veins (red). The $570 \mathrm{~nm}$ early-phase (circles) and peak-phase (dashed circles) RO (filled arrowheads) and dilated arterioles (open arrowheads). Orien-

time $x$, respectively. The scalars $\alpha_{1}$ and $\alpha_{2}$ are determined by stimulus intensity and ISI.

\section{0 and $610 \mathrm{~nm}$ signal etiology}

Origins of the optical signals differ according to wavelength range and event-related timing. In addition, signals acquired at a specific wavelength should be a mixture derived from several etiologies. Here, we reconfirm how 570 and $610 \mathrm{~nm}$ optical signals are related to fundamental physiological parameters.

$570 \mathrm{~nm}$ signals. A recent study of reflected light spectra from a brain tissue simulating phantom and transmitted light spectra in clear hemoglobin solution showed that optical signals near $569 \mathrm{~nm}$ are independent of hemoglobin oxygen saturation (Sato et al., 2002). When $\Delta$ ratio $_{\lambda}$ changes from 0 to -0.05 (the typical size of a $570 \mathrm{~nm}$ response), $\Delta$ absorbance $_{\lambda}\left[\log \left\{I_{\lambda}\right.\right.$ base $\left.\left./ I_{\lambda}(t)\right\}\right]$ changes from 0 to 0.022 . In this response range, $\Delta$ ratio $_{\lambda}$ and $\Delta$ absorbance $_{\lambda}$ are almost proportional; the ratio between them varies by only $2.6 \%$. If we assume the light attenuation and optical path length distribution caused by light scattering and the ratio of $\Delta \mathrm{ab}-$ 
A

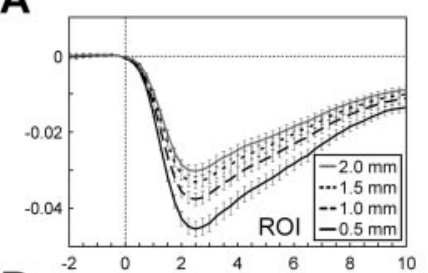

B

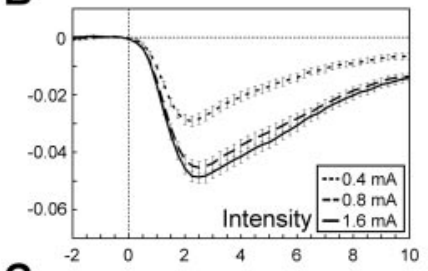

C
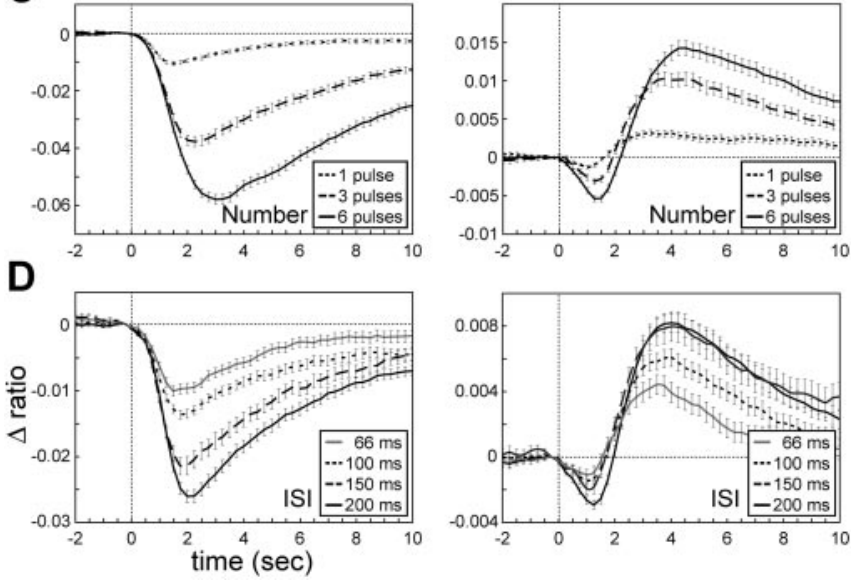

Figure 2. Time courses of optical intrinsic signals at 570 and $610 \mathrm{~nm}$ averaged across nine rats. Error bars indicate $S E M s$. $A$, Dependence of optical signal magnitude on $R O$ I size in response to the standard stimulation ( $0.8 \mathrm{~mA}, 5$ pulses, $200 \mathrm{msec} \mid S \mathrm{SI}) . B-D$, Dependence of optical signal magnitude on variable stimulus intensity (protocol 1 ) measured in the $0.5 \mathrm{~mm} \mathrm{ROI}(B)$, on variable stimulus number (protocol $2 ; C$ ), and on variable ISI (protocol $3 ; D$ ).

sorbance $_{570}$ to $\Delta$ ratio $_{570}$ change little, then $\Delta$ ratio $_{570}$ approximates changes in total hemoglobin concentration (Sheth et al., 2004). On a microcirculatory scale, curvilinear $570 \mathrm{~nm}$ signals would represent an increase in volume of the axial red blood cell (RBC) core caused by pial arteriole dilatation (McHedlishvili, 1991). Diffuse signals in the capillary bed may originate from an increase in capillary hematocrit such as RBC linear density (Johnson et al., 1971; Hudetz et al., 1999), capillary expansion, or RBC recruitment to poorly perfused capillaries (Villringer et al., 1994).

Equating changes in hemoglobin concentration with changes in CBV assumes a constant hematocrit, which may not hold true during functional activation. In a strict sense, hematocrit varies in arterial, capillary, and venous compartments in response to changes in physiological state (Johnson et al., 1971; McHedlishvili, 1991). Some studies compared both optical intrinsic signals and CBV measured using intravascular dyes distributed in plasma (Frostig et al., 1990; Narayan et al., 1995). They found similar localization or signal intensity time courses, supporting the use of $570 \mathrm{~nm}$ signals as an indicator of CBV. However, there seem to be differences in the time courses between total hemoglobin concentration measured by diffuse optical tomography and cerebral plasma volume measured by MRI using an exogenous contrast agent (Siegel et al., 2003).

$610 \mathrm{~nm}$ signals. Several investigators have suggested that a transient tissue deoxygenation evoked by neural activity ("initial dip") is responsible for early negative changes at $610 \mathrm{~nm}$ (Malonek and Grinvald, 1996; Nemoto et al., 1999; Mayhew et al., 2000; Jones et al., 2001; Sato et al., 2002), but the existence of the dip is still under debate (Lindauer et al.,
2001, 2003). Although deoxyhemoglobin is the most dominant absorbing species in this wavelength range, oxyhemoglobin also absorbs $610 \mathrm{~nm}$ light with approximately one-third the absorption coefficient in a braintissue-simulating phantom (Sato et al., 2002). Thus, a rapid increase in oxyhemoglobin with little change in deoxyhemoglobin might generate the decrease in $610 \mathrm{~nm}$ reflectance. Multicomponent spectrophotometric analysis showed that the oxyhemoglobin component contributes more significantly in the latter half of the early negative phase (Nemoto et al., 1999; Sato et al., 2002). In addition, arteriolar dilatation reduces $600 \mathrm{~nm}$ range light reflectance (Chen-Bee et al., 1996; Nemoto et al., 1999).

Based on the spatiotemporal dynamics and wavelength dependence of optical signals, we assume that the $610 \mathrm{~nm}$ initial negative signal is determined mainly by a balance of the following: (1) an increase in deoxyhemoglobin, which starts from venous-side capillaries in the activated neural tissue and moves convergently to the draining venules, caused by an increase in the conversion of oxyhemoglobin to deoxyhemoglobin associated with an increase in local oxygen tension gradients (Vanzetta and Grinvald, 1999; Ances et al., 2001; Masamoto et al., 2003; Thompson et al., 2003); (2) an increase in oxyhemoglobin (after a vascular response delay), which starts from arterial-side capillaries in response to microvascular flow control structures (Harrison et al., 2002) and propagates retrograde to proximal arterioles (Iadecola et al., 1997); and (3) a reduction in deoxyhemoglobin (after a flow transit time delay, and caused by an overcompensatory blood flow increase), which starts from venousside capillaries near the activated neural tissue and spreads over the vascular response area. Thus, in the latter half of the early negative response, the magnitude and distribution of $610 \mathrm{~nm}$ signals may depend on vascular responses that do not exactly reflect neural activity (Nemoto et al., 1999; Chen-Bee et al., 2000; Sato et al., 2002).

\section{Results}

We observed functional signal-dependent differences in spatiotemporal evolution patterns and linear relationships with $\Sigma F P$. Optical intrinsic signals of $570 \mathrm{~nm}$ centered on activated pial arterioles and demonstrated similar spatial geometry at different time points and across graded stimulation. The spatial pattern of $610 \mathrm{~nm}$ signals metamorphosed over time from the capillary bed to large draining veins. Correlation analyses revealed significant linear relationships between $\Sigma F P$ and both 570 and $610 \mathrm{~nm}$ (early dip and late positive) signals. On a trial-by-trial basis, the determination coefficients of $570 \mathrm{~nm}$ signals were considerably higher than those of $610 \mathrm{~nm}$ signals, and $570 \mathrm{~nm}$ signals even correlated with minute $\Sigma$ FP fluctuations within trials of the same stimulus condition.

We also found stimulus paradigm-dependent differences in neuronal-hemodynamic linearity. Although 570 and $610 \mathrm{~nm}$ late positive signals increased linearly with $\Sigma$ FP while varying stimulus number, they exhibited a threshold and steeper gradient while varying stimulus intensity. This functional paradigm dependence caused nonlinear data scattering in the overall data analyses.

\section{ROI and microelectrode locations}

The time courses of optical signal responses to a standard stimulation ( $0.8 \mathrm{~mA}, 200 \mathrm{msec}$ ISI, five pulses) showed similar patterns across all subjects. We observed a monophasic decrease in reflected light intensity at $570 \mathrm{~nm}$ and biphasic changes at $610 \mathrm{~nm}$ (an early decrease and late increase). We determined eight maximum-response ROIs (four sizes, two time epochs) in the 570 and $610 \mathrm{~nm} \Delta$ ratio images, respectively. Figure 1 shows optical signals and ROI locations in a representative case. At $570 \mathrm{~nm}$, ROI locations almost overlapped between the early and peak phases (Fig. $1 E$ ). In contrast, at $610 \mathrm{~nm}$, ROI locations differed markedly between the early negative and late positive-peak phase, with the late-phase ROI shifted toward a large draining vein (Fig. 
$1 G)$. The locations of early-phase 570 and early-phase $610 \mathrm{~nm}$ ROIs overlapped (Fig. $1 F$ ), but the smaller ROIs deviated to an activated arteriole at $570 \mathrm{~nm}$ and a venule at $610 \mathrm{~nm}$ (Fig. $1 F)$.

Across nine subjects, the average center-to-center distance for all four ROIs between the $570 \mathrm{~nm}$ early and peak phases was $158 \pm 190 \mu \mathrm{m}$ (mean $\pm \mathrm{SD} ; n=36$ ROIs). The distance between the $610 \mathrm{~nm}$ early negative and late positive-peak phases was $930 \pm 352 \mu \mathrm{m}$. The distance between the $570 \mathrm{~nm}$ early and $610 \mathrm{~nm}$ early phases was $196 \pm 147 \mu \mathrm{m}$. The corresponding $\mathrm{ROI}$ spatial overlap rates were 78,20 , and $74 \%$, respectively.

We used early-phase ROIs at 570 and $610 \mathrm{~nm}$ for later analyses, because ROIs of the $610 \mathrm{~nm}$ early negative and $570 \mathrm{~nm}$ early and peak phases significantly overlapped. The average distances between the microelectrode locations and the four ROI centers in the early phase were $651 \pm 198$ $\mu \mathrm{m}$ at $570 \mathrm{~nm}$ and $654 \pm 220 \mu \mathrm{m}$ at 610 $\mathrm{nm}$, an insignificant difference.

\section{Temporal dynamics of optical signal magnitudes}

The time courses of optical signals showed similar patterns despite changes in stimulus intensity, number, and ISI. Figure $2 \mathrm{~A}$ shows the dependence of optical signal magnitudes on ROI size in response to the standard stimulation across nine subjects. The magnitudes were larger in smaller ROIs, especially at $570 \mathrm{~nm}$. Figure $2 B-D$ shows average time courses of optical signals in the $0.5 \mathrm{~mm}$ ROIs across nine subjects, indicating that the response magnitudes at both wavelengths increased with stronger intensity, greater number of pulses, and longer ISI within the range of stimulus parameters tested. In the $610 \mathrm{~nm}$ responses, the magnitude ratio of the early negative to the late positive peak was $0.33 \pm$ 0.06 (mean $\pm \mathrm{SD} ; n=10$ ) across all stimulation parameters.

We also calculated the functional CNR of 570 and $610 \mathrm{~nm}$ signals for the $0.5 \mathrm{~mm}$ ROI time series by dividing the peak response magnitude by the time-dependent SD of prestimulus baseline fluctuations. CNR values of 570 and $610 \mathrm{~nm}$ early and late responses to the standard stimulation were $5.4 \pm 0.6,1.8 \pm$ 0.8 , and $2.9 \pm 1.1$ ( $n=4$ subjects $)$, respectively. We also performed a power spectral density analysis of $10 \mathrm{sec}$ baseline data to investigate the origin of the noise fluctuations. We found a peak in power at $\leq 0.1 \mathrm{~Hz}$ and a $1-2 \mathrm{~Hz}$ narrow band with associated harmonics related to respiration. These noise fluctuations arose from physiological processes and not from optical measurement systems. We did not detect any significant signals related to preceding functional activity in the prestimulus baseline period.

\section{Spatial localization and evolution of optical signals}

Figure 3 illustrates spatiotemporal dynamics of typical optical signals revealed by contour maps of the absolute signal intensities and their horizontal and vertical peak cross sections. In $570 \mathrm{~nm}$ contour maps and cross sections (Fig. $3 A-D$ ), the peak and ridge of signals originated from pial arteries, and the valley corresponded to pial veins. The arrangement of these vessels determined the overall response shape. The spatial profiles of peakphase $(t=1.75-2.25 \mathrm{sec}) 570 \mathrm{~nm}$ signals evoked by two-pulse stimulation were similar to those of the early phase $(t=1-1.5 \mathrm{sec})$ evoked by five-pulse stimulation (Fig. $3 B, D$ ). These cross sections and contour maps, transformed by a geometric scaling factor (Fig. $3 C, D$ ), appeared similar to those at the peak phase evoked by five-pulse stimulation (Fig. $3 A$ ).

The peak of $610 \mathrm{~nm}$ signals originated from pial veins (Fig. $3 E-H)$. The spatial profiles in the latter part of the early negative phase $(t=1.5-2 \mathrm{sec})$ and late positive-peak phase $(t=4.5-5 \mathrm{sec})$ were strongly distorted by large activated veins (Fig. $3 F, G$ ). In contrast, in the initial part of the early negative phase $(t=1-1.5$ $\mathrm{sec}$ ), signals were distributed over capillary beds with less conspicuous venous signals, and contour lines and cross sections were gently sloping and concentric (Fig. $3 E$ ). Signals from pial arterioles showed negative directional changes in all phases (Fig. $3 E, G$, red dashed lines).

Thus, the spatial profiles of $610 \mathrm{~nm}$ signals metamorphosed 
A
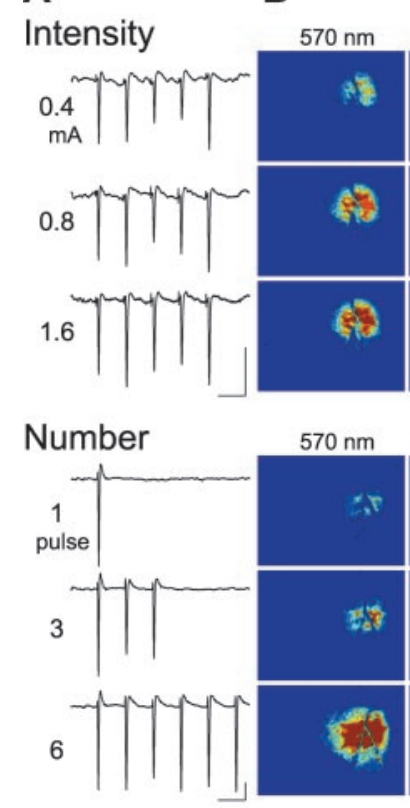

ISI
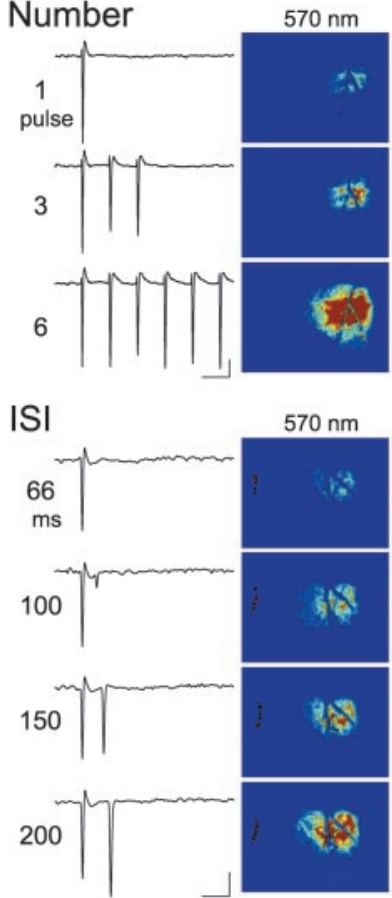

$570 \mathrm{~nm}$
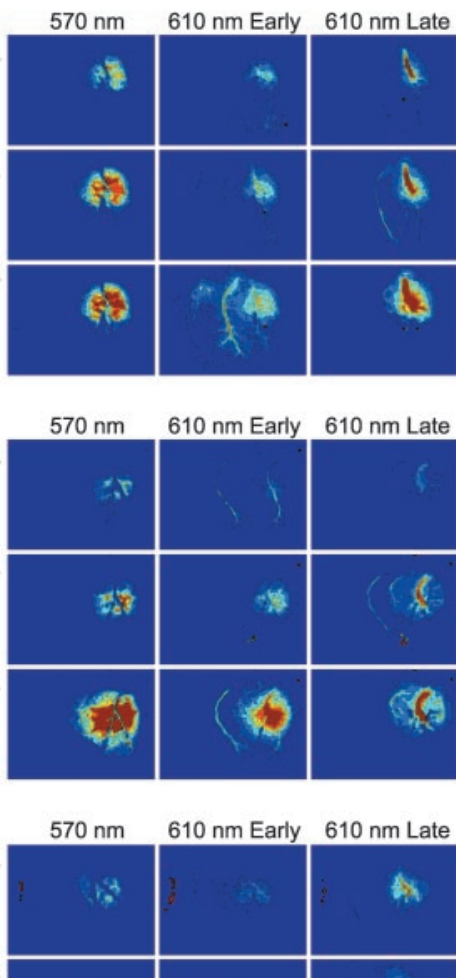

C
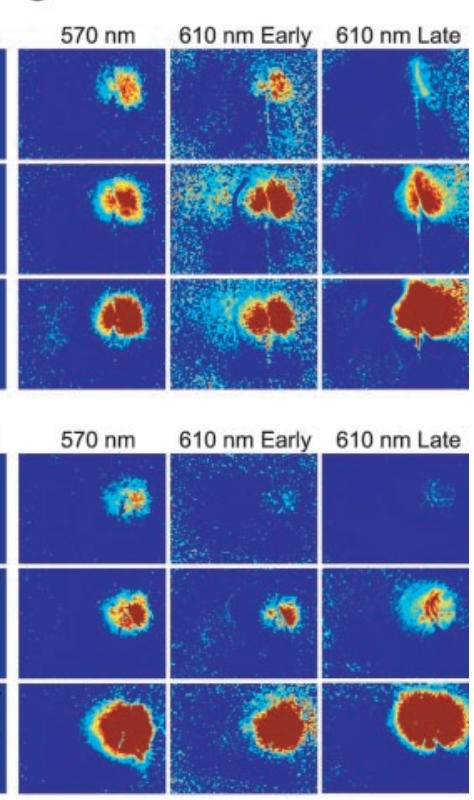

$610 \mathrm{~nm}$ Late
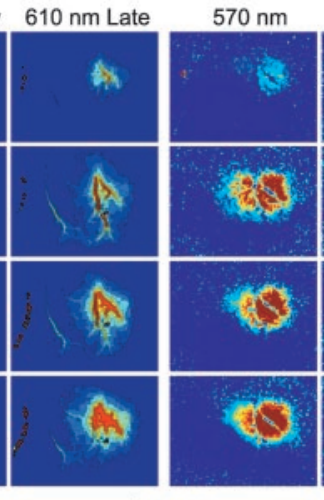

$610 \mathrm{~nm}$ Early $610 \mathrm{~nm}$ Late

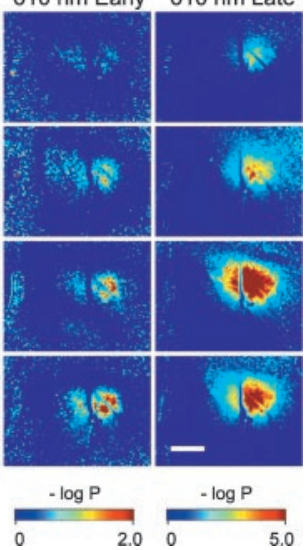

ond pulse with $200,150,100$, and $66 \mathrm{msec}$ ISI were $121 \pm 29,66 \pm 34,24 \pm 15$, and $11 \pm 2 \%$ of the first response amplitude, respectively (mean $\pm \mathrm{SD} ; n=9$ subjects). The second responses were strongly inhibited with an ISI of $<100-150 \mathrm{msec}$. Because a 200 msec ISI was sufficient for recovery of the evoked FP amplitude in the present experiment, protocol 1 modulated $\Sigma F P$ with changes in the amplitude of an individual FP (evoked by a single stimulus) at the same rate; in contrast, protocol 2 cumulatively modulated $\Sigma$ FP without changing individual FP amplitude. Protocol 3 modulated $\Sigma F P$ with changes in the second FP amplitude.

Consequently, the stimulus paradigm of protocol 1 tested the homogeneity property of linear systems, protocol 2 tested the additivity property, and protocol 3 tested combined homogeneity and additivity properties (i.e., the superposition property).

\section{Areal extent of optical signals}

Figure 4 shows representative cases of evoked FP and areal extent quantified using both absolute signal intensity and statistical thresholding. The areal extent of 570 and $610 \mathrm{~nm}$ signals increased with increasing stimulus intensity, number, and ISI. Using identical thresholds, the areal extent of 570 and $610 \mathrm{~nm}$ late peak signals was far larger than that of $610 \mathrm{~nm}$ early peak signals. Importantly, pixels in pial vessels with the highest $\Delta$ ratio value in the absolute threshold map (Fig. 4B) frequently showed relatively lower $p$ value than pixels in adjacent cortical areas in the $p$ value map (Fig. 4C). Inversely, cortical areas with low $\Delta$ ratio value in the absolute threshold map showed relatively higher significance in the $p$ value map. This finding suggests that larger signal fluctuations existed in pial vessels than in the capillary bed, resulting in less vascular contribution to the $p$ value map.

over time, whereas $570 \mathrm{~nm}$ signals evolved with spatial similarity across time and graded stimulation. The peak of $610 \mathrm{~nm}$ signals shifted from the capillary bed to the surrounding large draining veins, whereas the $570 \mathrm{~nm}$ peak remained at the central activated pial arteriole.

\section{Evoked field potentials}

In response to the standard stimulation $(0.8 \mathrm{~mA}, 200 \mathrm{msec}$ ISI, five pulses), the average amplitude of the negative component was $2.61 \pm 0.05 \mathrm{mV}$ (mean $\pm \mathrm{SD} ; n=5$ pulses in all subjects) (Fig. 4A). There was no significant difference between the amplitudes of the first response and subsequent responses in averaged data. In protocol 1 (variable stimulus intensity), the amplitudes of responses to 0.4 and $1.6 \mathrm{~mA}$ stimulation were $80 \pm 11$ and $106 \pm 7 \%$ of the $0.8 \mathrm{~mA}$ standard response, respectively. In protocol 3 (variable ISI), the amplitudes of the responses to the sec-

\section{Correlation analyses on averaged data}

Figure 5, $A$ and $B$, shows relationships between $\Sigma F P$ and peak $\Delta$ ratio response magnitudes in all four ROIs using both raw and normalized average data from nine subjects. In the raw data (Fig. $5 A$ ), the data points and regression lines were segregated by ROIs. In the normalized data, however, they largely overlapped (Fig. $5 B$ ). These results confirmed that there were similar strong linear relationships between average $\Sigma F P$ and 570 and $610 \mathrm{~nm}$ (both early and late) peak response magnitudes despite ROI size differences.

Figure 5, $C$ and $D$, shows relationships between $\Sigma F P$ and areal extent quantified with three absolute and statistical thresholds. We observed positive correlations between $\Sigma F P$ and 570 and 610 $\mathrm{nm}$ peak response areal extents despite differences in thresholds and quantification methods. 


\section{Correlation analyses on individual trial data}

We also examined these correlative relationships on a trial-by-trial basis for all nine subjects. We had previously found positive correlations between $\Sigma F P$ and 570 and $610 \mathrm{~nm}$ late peak response magnitudes on a trial-by-trial basis in individual subjects. To investigate the universality of the principle observed in individual subjects, we used normalized data because of considerable variations in the regression line slope across subjects. Figure 6 shows scatter plots of normalized $\Sigma$ FPs versus 570 and $610 \mathrm{~nm}$ peak response magnitudes in $0.5 \mathrm{~mm}$ ROIs in the 1305 trials. Linear regression analyses indicated significant positive correlations between normalized $\Sigma F P s$ and 570 and $610 \mathrm{~nm}$ (both early and late) responses in all stimulation protocols. The determination coefficients $\left(r^{2}\right)$ of $570 \mathrm{~nm}$ responses were considerably higher than those of $610 \mathrm{~nm}$ early and late responses in all stimulation protocols. The $r^{2}$ values were also higher in protocol 2 (variable stimulus number) than in the other stimulation protocols. These findings were consistent across all four ROI sizes, and regression lines from each ROI overlapped one another as well as normalized average data.

Within trials of the same stimulus condition, we observed $\Sigma F P$ fluctuations that might be caused by internal processes of neural circuitry but not by external stimulation, especially in low-intensity stimuli (e.g., $0.4 \mathrm{~mA}$, five pulses). We examined how accurately optical signals reflected these small $\Sigma$ FP fluctuations. Figure $7 A-C$ shows scatter plots of normalized $\Sigma F P$ versus optical responses in typical trial sets of protocol 1 , clearly revealing the correlative difference between 570 and $610 \mathrm{~nm}$ early and late signals. Figure $7 D$ shows the time series of 15 trial data in response to the standard stimulation, indicating that $570 \mathrm{~nm}$ signals varied in correspondence with $\Sigma F P$ fluctuations. Figure $7 E$ shows the distribution of $r^{2}$ values in 27 trial sets of protocol 1. These data further support the notion that $570 \mathrm{~nm}$ signals reflected intrinsic ¿FP fluctuations more accurately than did $610 \mathrm{~nm}$ signals.

We also observed apparently contradictory findings in $610 \mathrm{~nm}$ signals. In averaged data, peak magnitudes of early negative responses correlated positively with peak magnitudes of late positive responses (Fig. 7F, G, insets). However, the trial-by-trial analysis shows that they did not correlate overall and correlated negatively within the same stimulus trials (Fig. 7F,G).

\section{Nonlinearities in optical signal responses}

Importantly, the regression line slopes in protocol 1 (variable stimulus intensity) were steeper than those in protocol 2 and did not pass through the origin (Figs. $6 A, B, 8 A$ ). We tested the difference between regression line slopes with a Student's $t$ test, which indicated a significant difference in $570 \mathrm{~nm}\left(p<10^{-27}\right)$ and $610 \mathrm{~nm}\left(p<10^{-8}\right)$ late positive signals but insignificance in $610 \mathrm{~nm}$ early negative signals ( $p=0.53$ ) between different protocols. The two significantly different regression lines indicate that stimuli eliciting the same $\Sigma F P$ may produce hemodynamic responses of different magnitude. For example, frequent but small asynchronous neuronal population activity may produce hemodynamic responses with different magnitudes compared with less frequent but large synchronous neuronal population activity. Conversely, equal-magnitude hemodynamic responses may be observed by stimuli producing different patterns of neuronal activity.

We also determined the best-fitting geometric curves $(Y=$ $\left.a X^{b}\right)$ from both protocol 1 and protocol 2 data sets in $570 \mathrm{~nm}$ signals (Fig. 8A). The protocol 1 equation of the geometric curve was $Y=1.050 X^{0.967}$ (95\% confidence interval for the power: $0.899<b<1.034)$, whereas the protocol 2 equation was $Y=$ $0.091 X^{2.427}$ (95\% confidence interval: $2.183<b<2.670$ ), suggesting a nonlinear relationship in variable stimulus intensity 


\section{$570 \mathrm{~nm}$}

A Intensity
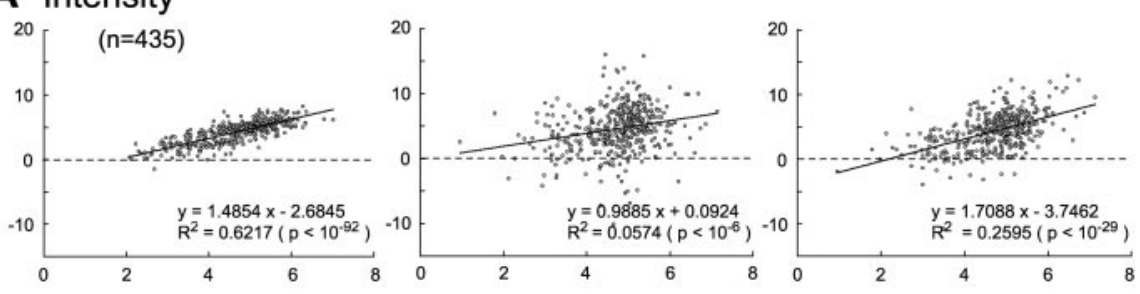

B Number
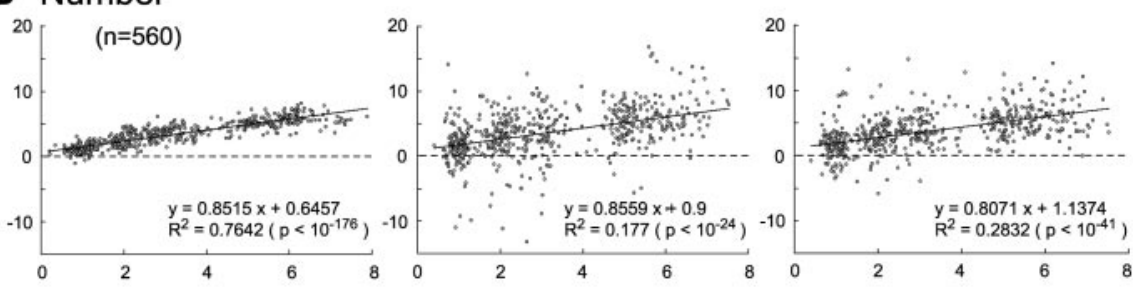

C ISI
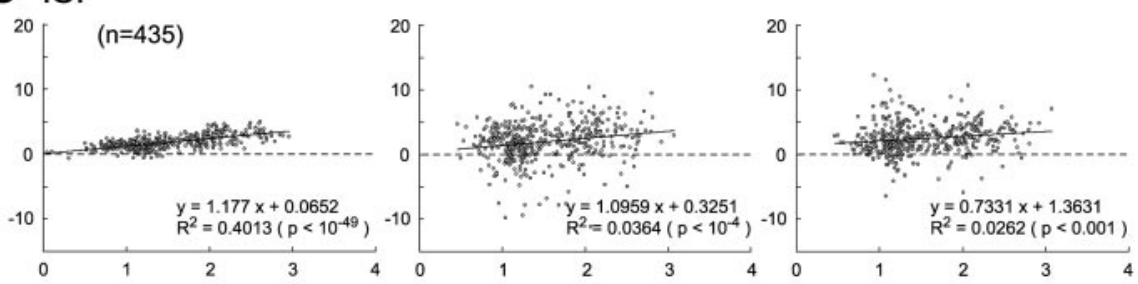

D Total
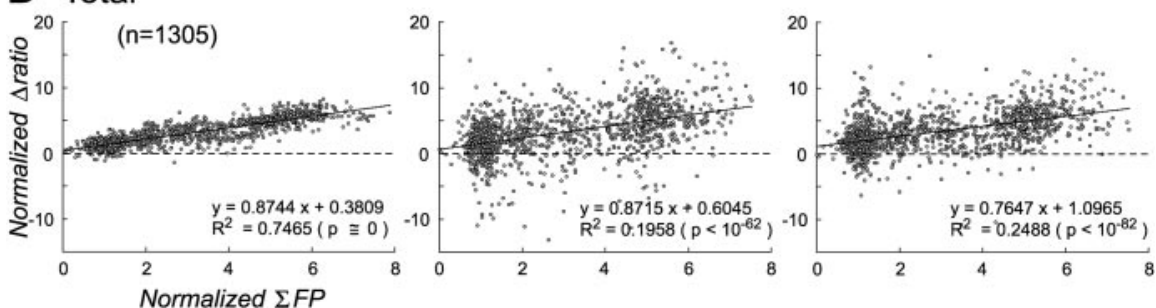

Figure 6. Correlation analyses between normalized $\Sigma F P$ and optical signal magnitude based on individual trial data from nine subjects. $A-D$, Scatter plots of normalized $\sum F P$ versus $570 \mathrm{~nm}, 610 \mathrm{~nm}$ early-negative, and $610 \mathrm{~nm}$ late-positive peak signal responses. $A$, Protocol 1 , variable stimulus intensity. $B$, Protocol 2 , variable stimulus number, including the data from $0.8 \mathrm{~mA}$, five pulse, $200 \mathrm{msec}$ ISI stimulation (protocol 1) and $0.8 \mathrm{~mA}$, two pulse, $200 \mathrm{msec}$ ISI stimulation (protocol 3). C, Protocol 3, variable ISI. $D$, Total trial data correlations $(n=1305)$.

trials. The diversity of these responses caused nonlinear data scattering in the overall data analyses (Figs. $6 D, 8 \mathrm{~A}$ ).

Figure $8 B$ shows a simple model reached from protocols 1 and 2 and some additional data. If $\Sigma F P$ is constant, the hemodynamic responses increase with increasing average FP amplitude (i.e., $\Sigma$ FP divided by stimulus number) (Fig. $8 B, P_{4}-P_{7}$ ). This pattern suggests that spatiotemporal coherence in neuronal population activity, which is not captured by the single-input parameter $\Sigma F P$, is possibly involved in hemodynamic response formation.

\section{Discussion}

The linear relationships between $\Sigma$ FPs and optical intrinsic signals were critically dependent on functional signal etiology (blood volume vs oxygenation) and paradigm. Recent studies have demonstrated that functional blood flow and BOLD responses are better correlated with local FP than single- and multiple-unit activity (Mathiesen et al., 1998; Logothetis et al., 2001), suggesting that input activity (presynaptic and postsynaptic) plays a more important role in mediating hemodynamic responses than output spiking activity (Lauritzen, 2001; Logothetis,
2002). We verified the linear correlations between $\Sigma$ FPs and metabolic and hemodynamic signals, but the determination coefficients were considerably different between blood volume and oxygenation signals, and the degrees of linearity were significantly different between conditions for which stimulus intensity and number (i.e., challenging homogeneity and additivity properties of the system) were varied.

\section{Spatiotemporal characteristics of CBV signals}

The spatiotemporal dynamics of $570 \mathrm{~nm}$ signals exhibited several interesting features. First, although the center-to-center distance between early negative and late positive $610 \mathrm{~nm}$ signals was nearly $1 \mathrm{~mm}$, the distance between early 570 and $610 \mathrm{~nm}$ signals and between $570 \mathrm{~nm}$ early-phase and peak-phase signals was $\sim 200 \mu \mathrm{m}$. Second, although the spatial profile of 610 $\mathrm{nm}$ signals changed over time, $570 \mathrm{~nm}$ signals evolved with spatial similarity across time and graded stimulation. These findings may reflect the relationship between anatomical-functional organizations of microvascular modules and activated neuronal units (Woolsey et al., 1996). Furthermore, changes in pial arteriolar diameter lagged behind changes in capillary hemoglobin concentration, especially in the later phase (Fig. $1 B$ ), suggesting the existence of fine flow control systems in intraparenchymal microvessels (Harrison et al., 2002). Lastly, the majority of $570 \mathrm{~nm}$ signals came from pial arterioles and the capillary bed rather than pial venules (Figs. $1 B, 3 A-D)$. This observation is consistent with findings in the cerebellar cortex and measurements of relative arterial and venous blood volume changes induced by neuronal activation and hypercapnia (Iadecola et al., 1997; Lee et al., 2001). In brief stimulus paradigms, these data support the hypothesis that the functional CBV increase predominantly occurs in arterial and capillary compartments rather than venous compartments (Lee et al., 2001), which differs from the balloon model hypothesis that functional CBV increases occur mainly in veins (Buxton et al., 1998).

\section{CBV signal response linearity}

We found a significant linear relationship between $\Sigma$ FP and 570 $\mathrm{nm}$ signals, with determination coefficients $\left(r^{2}\right)$ considerably higher than those of $610 \mathrm{~nm}$ signals and overlapping regression lines from four ROIs. These results suggest the following implications: (1) During brief ( $\leq 1 \mathrm{sec}$ ) repetitive stimulation, $570 \mathrm{~nm}$ responses consist of a simple summation of individual unit responses (Martindale et al., 2003). (2) The difference between $r^{2}$ values of $570 \mathrm{~nm}$ signals and $610 \mathrm{~nm}$ early and late signals may be attributable to differences in both CNR and the contribution of nonlinear components inherent in these signals. Whereas $570 \mathrm{~nm}$ signals are attributable to the functional regulation of CBF and 
$\mathrm{CBV}, 610 \mathrm{~nm}$ signals reflect the balance of oxygen extraction, $\mathrm{CBF}$, and $\mathrm{CBV}$, which may introduce complicated departures from linearity. These nonlinearities may explain the discrepancies between studies measuring CBF and BOLD signals (Rees et al., 1997; Miller et al., 2001). (3) The overlapping of regression lines from ROIs of different sizes suggests that the magnitude of signals at a distance from the activated center changes in response to different stimulations at a rate similar to those of signals in the activated center. In other words, these linear relationships might be macroscopically independent of ROI selection in a restricted region. This inference is consistent with the observation that $570 \mathrm{~nm}$ signals evolved with geometrical analogy across time and graded stimulation (Fig. 3A-D).

\section{Early oxygenation signals and neuronal activity}

Although it has been verified that early negative $610 \mathrm{~nm}$ signals or deoxygenation signals offer a small point-spread function and high spatial specificity to activated columnar structures (Frostig et al., 1990; Masino et al., 1993; Malonek and Grinvald, 1996; Thompson et al., 2003), it is unclear how accurately the response magnitude reflects the magnitude of evoked neural activity. The use of differential subtraction methods in optical imaging studies presupposes not only colocalization but also a linear relationship between optical signals and underlying neuronal activity. Polley et al. (1999) reported that the number of whisker deflections did not affect the area or peak magnitude of early negative $630 \mathrm{~nm}$ signals. We also observed inconsistent coupling between $\Sigma$ FPs and early negative $610 \mathrm{~nm}$ signals within and across subjects in hindpaw, forepaw, and whisker barrel cortex (Sheth et al., 2003). However, Peterson et al. (1998) found a near-linear relationship between extracellular spike count and monophasic negative $660 \mathrm{~nm}$ signals while varying vibrissal deflection angle. The present study shows that early negative $610 \mathrm{~nm}$ signals are positively correlated with $\Sigma$ FPs, but with a poor determination coefficient.

These conflicting results may be explained by differences in experimental procedures and species. For example, different anesthetic, surgical, and stimulation protocols could lead to different excitatory and inhibitory neuronal processes in thalamocortical and intracortical circuits (Simons and Carvell, 1989; CastroAlamancos and Oldford, 2002) and different hemodynamic responses. Moreover, the balance of $610 \mathrm{~nm}$ signal determinants (see Materials and Methods) can easily vary depending on the latency and magnitude of hemodynamic responses, which are significantly affected by manipulation of $\mathrm{P}_{\mathrm{CO}_{2}}$ (Harel et al., 2002) stimulus paradigm $(F, G)$.
B

\section{$610 \mathrm{~nm}$ Early}

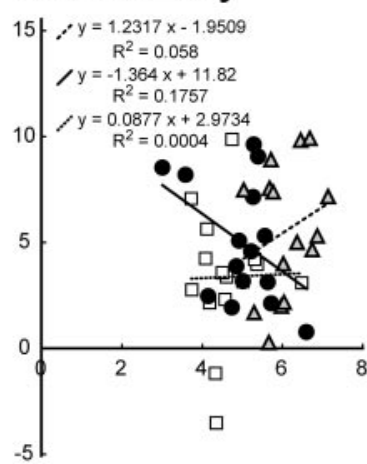

$610 \mathrm{~nm}$ Late

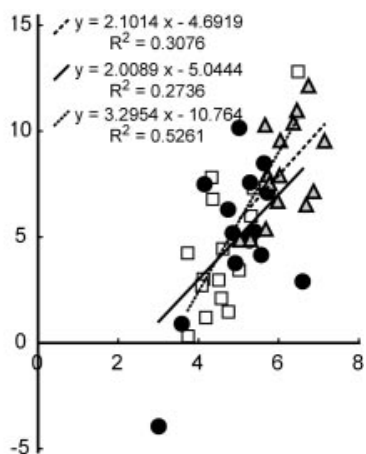

E

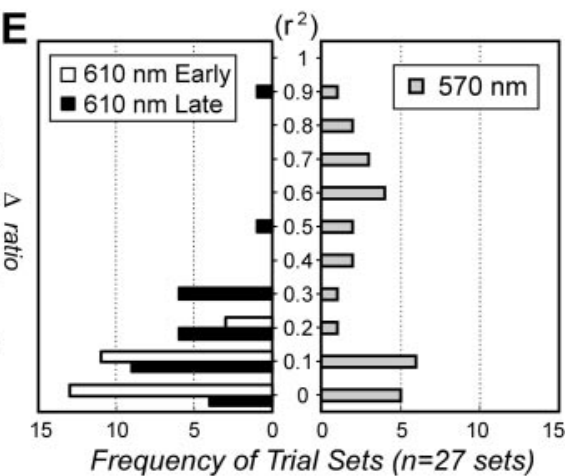

Frequency of Trial Sets ( $n=27$ sets)

$\mathbf{F}$

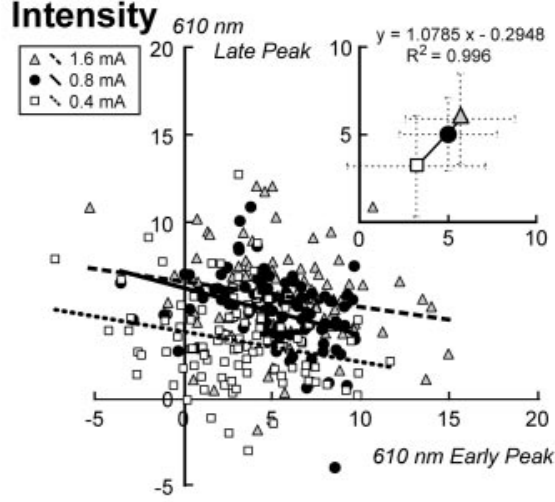

G

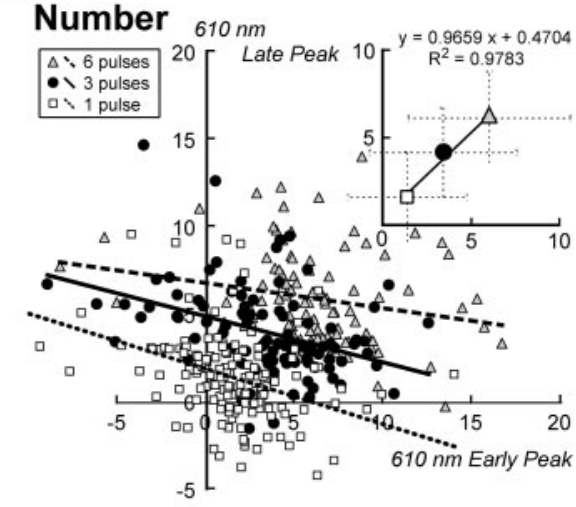

Figure 7. Trial-by-trial analyses of 570 and $610 \mathrm{~nm}$ early and late signals. A-C, Scatter plots of normalized $\Sigma F P$ and $570 \mathrm{~nm}$ peak $(A), 610 \mathrm{~nm}$ early-peak $(B)$, and $610 \mathrm{~nm}$ late-peak ( $C$ ) signals for protocol 1 in a representative case. D, Time series of $\Sigma F P$, 570 and $610 \mathrm{~nm}$ early-peak, and $610 \mathrm{~nm}$ late-peak signals for 15 trials of the standard stimulation (the same subject as in $A-C$ ). $E_{\text {, }}$ Distribution of determination coefficients $\left(r^{2}\right)$ in 27 trial sets of protocol 1 across nine rats shows the difference between the fidelity and $610 \mathrm{~nm}$ signals to intrinsic $\Sigma F P$ fluctuations within trials of the same stimulus condition. $F$, G, The relationships between $610 \mathrm{~nm}$ early and late peak responses in protocols $1(F)$ and $2(G)$. Although the average data evidenced positive linear correlations $(F, G$, inset), individual trial data showed no overall correlation and negative correlations within trials of the same

and $\mathrm{P}_{\mathrm{O}_{2}}$ (Lindauer et al., 2003). This inconsistency and apparent dependency on various physiological parameters raises concern as to whether the spatial profiles and magnitudes of early oxygenation signals reflect neuronal population activity per se.

\section{Late oxygenation signals}

Late positive $610 \mathrm{~nm}$ signals are analogous to positive BOLD fMRI signals, because both are associated with a decrease in deoxyhemoglobin (Ogawa et al., 1990). Peak responses were biased toward large draining veins, and spatial profiles were heavily influenced by venous structures (Fig. $3 F, G$ ). Similar findings have been observed in monkey and human optical imaging studies 
A

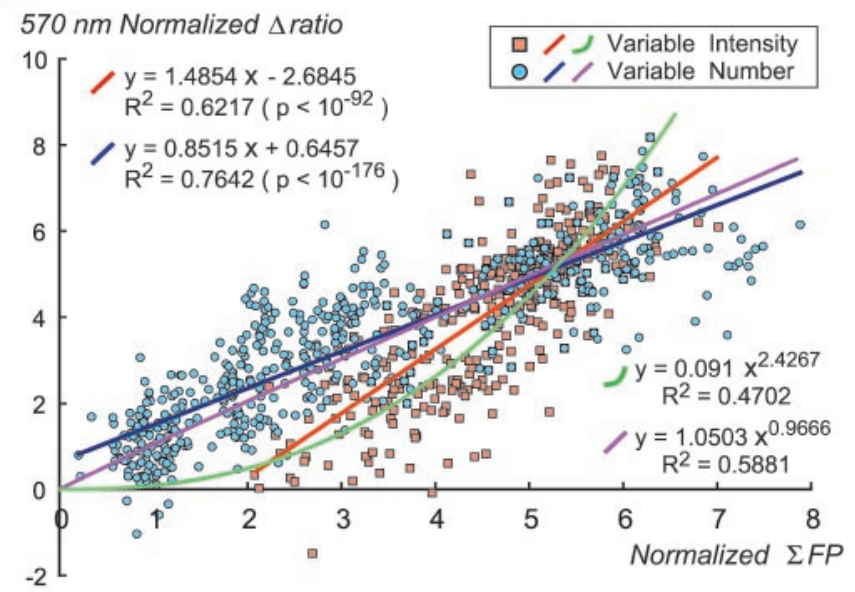

B

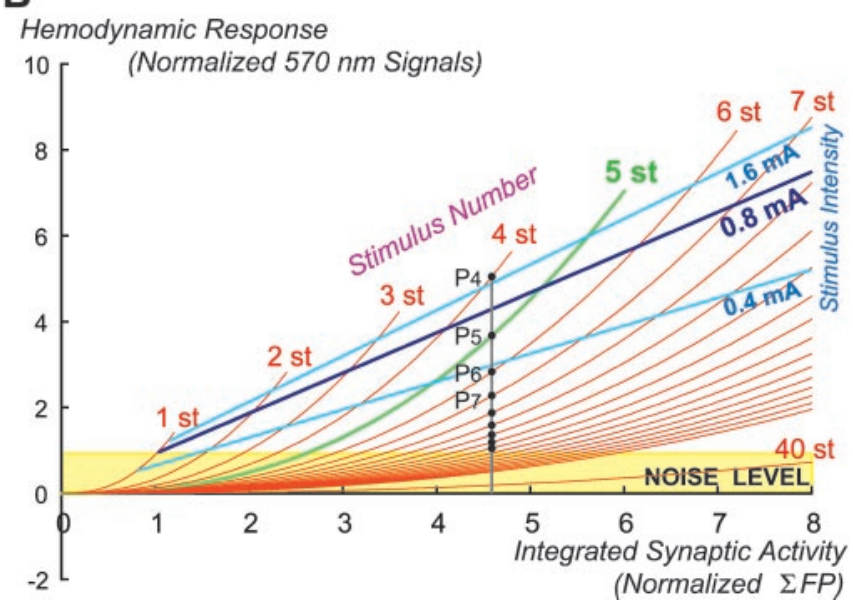

Figure 8. Stimulus paradigm dependence of linear relationships between $\Sigma F P$ and hemodynamic responses. A, Scatter plots of normalized $\Sigma F P$ versus $570 \mathrm{~nm}$ signal responses in the stimulus paradigms of protocols 1 and 2 . These relationships are approximated by straight lines and geometric curves and are compared between protocols. The slopes of regression lines were significantly different between protocols 1 and $2\left(p<10^{-27}\right.$; Student's $t$ test). The power for the best-fitting geometric curves in protocols 1 and 2 approximated 2.4 and 1 , respectively. $B, A$ simple model of the relationships between $\Sigma F P$ and hemodynamic responses in the stimulus paradigm with sufficient ISI for recovery of individual $\mathrm{FP}$ responses. The relationships are modeled by the following two equations: the linear equation $\left(Y=k_{1} X, k_{1}\right.$ is a function of stimulus intensity) for the paradigm of variable stimulus number, and the geometric curve equation $\left[Y=k_{2} X^{b}, k_{2}\right.$ is a function of stimulus number $(M)$, where $b=2.427$ and $k_{2}=0.091 \times$ $5^{1.427} \times \mathrm{N}^{-1.427}$ to fit the data sets as shown in $A$ ] for the paradigm of variable stimulus intensity. $P_{N}$ is a point on the geometric curve of stimulus number $N$ that falls on the same value of the $\Sigma F P$ axis.

(Shoham and Grinvald, 2001; Pouratian et al., 2002). Spatiotemporal dynamics (Fig. $3 E-H$ ) are also similar to BOLD responses described in the cat visual cortex (Duong et al., 2000). We found that $610 \mathrm{~nm}$ late positive signals also correlated linearly with ¿FPs. This result is consistent with other studies using optical imaging (Polley et al., 1999; Sheth et al., 2003) and fMRI (Arthurs et al., 2000; Ogawa et al., 2000; Logothetis et al., 2001).

\section{Early versus late oxygenation signals}

Although peak magnitudes of early negative responses correlated positively with those of late positive responses in averaged data, we found no correlation on a trial-by-trial basis and a negative correlation for trials in the same stimulus condition (Fig. $7 F, G$ ). To investigate these results, we performed a power spectral den- sity analysis on the $10 \mathrm{sec}$ of prestimulus baseline data. The power at $\sim 0.1 \mathrm{~Hz}$ most likely represents spontaneous flow oscillations (Hudetz et al., 1992; Mayhew et al., 1996). The magnitudes of the baseline fluctuations were similar to those of early negative responses. These findings suggest that large and slow baseline fluctuations influenced both early negative and late positive signals in the same direction, making them negatively correlated on a trialby-trial basis. Alternatively, the stochastic variations of hemodynamic responses may have affected the $610 \mathrm{~nm}$ early and late signals in the same direction. In the averaged data, however, the baseline fluctuations and hemodynamic response variations tended to cancel out, and the positive correlation reflected the fact that both individually correlated with $\Sigma$ FPs.

\section{Functional paradigm dependence of the linearity}

We observed a statistically significant departure from linearity in the relationships between neuronal and hemodynamic responses, depending on stimulus paradigm. The regression line slopes of 570 and $610 \mathrm{~nm}$ late signals in protocol 1 were steeper than those in protocol 2 and did not pass through the origin (Figs. $6 A, B, 8 A)$. This data distribution does not satisfy the requirement of homogeneity in linear systems, and implies either the existence of a hemodynamic response threshold (Nielsen and Lauritzen, 2001) or approximation by a geometric curve (Devor et al., 2003). The model shown in Figure 8 B may explain nonlinear components in paradigms modulating both FP response amplitude and total response number per unit time, such as paradigms that vary stimulus frequency (Ngai et al., 1999; Nielsen and Lauritzen, 2001; Sheth et al., 2003). Furthermore, simple subtractionbased analyses of hemodynamic signals produced by complex stimulus paradigms may not reflect a difference in $\Sigma$ FPs between paradigms. These nonlinearities associated with analysis procedures and stimulus paradigms have potentially profound implications for the interpretation of perfusion-based functional signals.

In conclusion, functional signal etiology (CBV vs oxygenation) dependence in spatiotemporal evolution patterns and linear relationships with $\Sigma$ FPs indicate that CBV-related hemodynamic signals have higher fidelity to integrated synaptic activity than oxygenation-derived signals and support the validity of applying linear systems analysis to $570 \mathrm{~nm}$ signals and analogs to evaluate neuronal activity. Functional paradigm dependence of the linearity associated with insufficiency of the homogeneity property will cause nonlinear data scattering in more complicated paradigms. This type of nonlinearity may reflect neglected participation of certain aspects of neuronal population activity that cannot be expressed by the single-parameter $\Sigma F P$, such as spatiotemporal coherence. Functional signal- and paradigm-dependent linearity and nonlinearity should be considered when interpreting hemodynamic signals and designing new experiments.

\section{References}

Ances BM, Buerk DG, Greenberg JH, Detre JA (2001) Temporal dynamics of the partial pressure of brain tissue oxygen during functional forepaw stimulation in rats. Neurosci Lett 306:106-110.

Arthurs OJ, Williams EJ, Carpenter TA, Pickard JD, Boniface SJ (2000) Linear coupling between functional magnetic resonance imaging and evoked potential amplitude in human somatosensory cortex. Neuroscience 101:803-806

Buxton RB, Wong EC, Frank LR (1998) Dynamics of blood flow and oxygenation changes during brain activation: the balloon model. Magn Reson Med 39:855-864.

Castro-Alamancos MA, Oldford E (2002) Cortical sensory suppression during arousal is due to the activity-dependent depression of thalamocortical synapses. J Physiol (Lond) 541:319-331.

Chen-Bee CH, Kwon MC, Masino SA, Frostig RD (1996) Areal extent quan- 
tification of functional representations using intrinsic signal optical imaging. J Neurosci Methods 68:27-37.

Chen-Bee CH, Polley DB, Brett-Green B, Prakash N, Kwon MC, Frostig RD (2000) Visualizing and quantifying evoked cortical activity assessed with intrinsic signal imaging. J Neurosci Methods 97:157-173.

Devor A, Dunn AK, Andermann ML, Ulbert I, Boas DA, Dale AM (2003) Coupling of total hemoglobin concentration, oxygenation, and neural activity in rat somatosensory cortex. Neuron 39:353-359.

Disbrow EA, Slutsky DA, Roberts TP, Krubitzer LA (2000) Functional MRI at 1.5 tesla: a comparison of the blood oxygenation level-dependent signal and electrophysiology. Proc Natl Acad Sci USA 97:9718-9723.

Duong TQ, Kim DS, Ugurbil K, Kim SG (2000) Spatiotemporal dynamics of the BOLD fMRI signals: toward mapping submillimeter cortical columns using the early negative response. Magn Reson Med 44:231-242.

Duong TQ, Kim DS, Ugurbil K, Kim SG (2001) Localized cerebral blood flow response at submillimeter columnar resolution. Proc Natl Acad Sci USA 98:10904-10909.

Friedberg MH, Lee SM, Ebner FF (1999) Modulation of receptive field properties of thalamic somatosensory neurons by the depth of anesthesia. J Neurophysiol 81:2243-2252.

Frostig RD, Lieke EE, Ts'o DY, Grinvald A (1990) Cortical functional architecture and local coupling between neuronal activity and the microcirculation revealed by in vivo high-resolution optical imaging of intrinsic signals. Proc Natl Acad Sci USA 87:6082-6086.

Harel N, Lee SP, Nagaoka T, Kim DS, Kim SG (2002) Origin of negative blood oxygenation level-dependent fMRI signals. J Cereb Blood Flow Metab 22:908-917.

Harrison RV, Harel N, Panesar J, Mount RJ (2002) Blood capillary distribution correlates with hemodynamic-based functional imaging in cerebral cortex. Cereb Cortex 12:225-233.

Heeger DJ, Ress D (2002) What does fMRI tell us about neuronal activity? Nat Rev Neurosci 3:142-151.

Hudetz AG, Roman RJ, Harder DR (1992) Spontaneous flow oscillations in the cerebral cortex during acute changes in mean arterial pressure. J Cereb Blood Flow Metab 12:491-499.

Hudetz AG, Wood JD, Biswal BB, Krolo I, Kampine JP (1999) Effect of hemodilution on RBC velocity, supply rate, and hematocrit in the cerebral capillary network. J Appl Physiol 87:505-509.

Iadecola C, Yang G, Ebner TJ, Chen G (1997) Local and propagated vascular responses evoked by focal synaptic activity in cerebellar cortex. J Neurophysiol 78:651-659.

Johnson PC, Blaschke J, Burton KS, Dial JH (1971) Influence of flow variations on capillary hematocrit in mesentery. Am J Physiol 221:105-112.

Jones M, Berwick J, Johnston D, Mayhew J (2001) Concurrent optical imaging spectroscopy and laser-Doppler flowmetry: the relationship between blood flow, oxygenation, and volume in rodent barrel cortex. NeuroImage 13:1002-1015.

Kim DS, Duong TQ, Kim SG (2000) High-resolution mapping of isoorientation columns by fMRI. Nat Neurosci 3:164-169.

Kim SG, Ugurbil K (2003) High-resolution functional magnetic resonance imaging of the animal brain. Methods 30:28-41.

Lauritzen M (2001) Relationship of spikes, synaptic activity, and local changes of cerebral blood flow. J Cereb Blood Flow Metab 21:1367-1383.

Lee SP, Duong TQ, Yang G, Iadecola C, Kim SG (2001) Relative changes of cerebral arterial and venous blood volumes during increased cerebral blood flow: implications for BOLD fMRI. Magn Reson Med 45:791-800.

Lindauer U, Royl G, Leithner C, Kuhl M, Gold L, Gethmann J, Kohl-Bareis M, Villringer A, Dirnagl U (2001) No evidence for early decrease in blood oxygenation in rat whisker cortex in response to functional activation. NeuroImage 13:988-1001.

Lindauer U, Gethmann J, Kuhl M, Kohl-Bareis M, Dirnagl U (2003) Neuronal activity-induced changes of local cerebral microvascular blood oxygenation in the rat: effect of systemic hyperoxia or hypoxia. Brain Res 975:135-140.

Logothetis NK (2002) The neural basis of the blood-oxygen-leveldependent functional magnetic resonance imaging signal. Philos Trans $\mathrm{R}$ Soc Lond B Biol Sci 357:1003-1037.

Logothetis NK, Pauls J, Augath M, Trinath T, Oeltermann A (2001) Neurophysiological investigation of the basis of the fMRI signal. Nature 412:150-157.

Malonek D, Grinvald A (1996) Interactions between electrical activity and cortical microcirculation revealed by imaging spectroscopy: implications for functional brain mapping. Science 272:551-554.
Mandeville JB, Marota JJ, Kosofsky BE, Keltner JR, Weissleder R, Rosen BR, Weisskoff RM (1998) Dynamic functional imaging of relative cerebral blood volume during rat forepaw stimulation. Magn Reson Med 39:615-624.

Martindale J, Mayhew J, Berwick J, Jones M, Martin C, Johnston D, Redgrave P, Zheng Y (2003) The hemodynamic impulse response to a single neural event. J Cereb Blood Flow Metab 23:546-555.

Masamoto K, Omura T, Takizawa N, Kobayashi H, Katura T, Maki A, Kawaguchi H, Tanishita K (2003) Biphasic changes in tissue partial pressure of oxygen closely related to localized neural activity in guinea pig auditory cortex. J Cereb Blood Flow Metab 23:1075-1084.

Masino SA, Kwon MC, Dory Y, Frostig RD (1993) Characterization of functional organization within rat barrel cortex using intrinsic signal optical imaging through a thinned skull. Proc Natl Acad Sci USA 90:9998-10002.

Mathiesen C, Caesar K, Akgoren N, Lauritzen M (1998) Modification of activity-dependent increases of cerebral blood flow by excitatory synaptic activity and spikes in rat cerebellar cortex. J Physiol (Lond) 512:555-566.

Mayhew J, Johnston D, Berwick J, Jones M, Coffey P, Zheng Y (2000) Spectroscopic analysis of neural activity in brain: increased oxygen consumption following activation of barrel cortex. NeuroImage 12:664-675.

Mayhew JE, Askew S, Zheng Y, Porrill J, Westby GW, Redgrave P, Rector DM, Harper RM (1996) Cerebral vasomotion: a $0.1-\mathrm{Hz}$ oscillation in reflected light imaging of neural activity. NeuroImage 4:183-193.

McHedlishvili G (1991) Dynamic structure of blood flow in microvessels. Microcirc Endothelium Lymphatics 7:3-49.

Miller KL, Luh WM, Liu TT, Martinez A, Obata T, Wong EC, Frank LR, Buxton RB (2001) Nonlinear temporal dynamics of the cerebral blood flow response. Hum Brain Mapp 13:1-12.

Moore CI, Nelson SB, Sur M (1999) Dynamics of neuronal processing in rat somatosensory cortex. Trends Neurosci 22:513-520.

Narayan SM, Esfahani P, Blood AJ, Sikkens L, Toga AW (1995) Functional increases in cerebral blood volume over somatosensory cortex. J Cereb Blood Flow Metab 15:754-765.

Nemoto M, Nomura Y, Sato C, Tamura M, Houkin K, Koyanagi I, Abe H (1999) Analysis of optical signals evoked by peripheral nerve stimulation in rat somatosensory cortex: dynamic changes in hemoglobin concentration and oxygenation. J Cereb Blood Flow Metab 19:246-259.

Ngai AC, Jolley MA, D’Ambrosio R, Meno JR, Winn HR (1999) Frequencydependent changes in cerebral blood flow and evoked potentials during somatosensory stimulation in the rat. Brain Res 837:221-228.

Nicolelis MA, Fanselow EE (2002) Thalamocortical [correction of Thalamcortical] optimization of tactile processing according to behavioral state. Nat Neurosci [Erratum (2002) 5:704] 5:517-523.

Nielsen A, Lauritzen M (2001) Coupling and uncoupling of activitydependent increases of neuronal activity and blood flow in rat somatosensory cortex. J Physiol (Lond) 533:773-785.

Ogawa S, Lee TM, Kay AR, Tank DW (1990) Brain magnetic resonance imaging with contrast dependent on blood oxygenation. Proc Natl Acad Sci USA 87:9868-9872.

Ogawa S, Lee TM, Stepnoski R, Chen W, Zhu XH, Ugurbil K (2000) An approach to probe some neural systems interaction by functional MRI at neural time scale down to milliseconds. Proc Natl Acad Sci USA 97:11026-11031.

Peterson BE, Goldreich D, Merzenich MM (1998) Optical imaging and electrophysiology of rat barrel cortex. I. Responses to small single-vibrissa deflections. Cereb Cortex 8:173-183.

Polley DB, Chen-Bee CH, Frostig RD (1999) Varying the degree of singlewhisker stimulation differentially affects phases of intrinsic signals in rat barrel cortex. J Neurophysiol 81:692-701.

Pouratian N, Sicotte N, Rex D, Martin NA, Becker D, Cannestra AF, Toga AW (2002) Spatial/temporal correlation of BOLD and optical intrinsic signals in humans. Magn Reson Med 47:766-776.

Rees G, Howseman A, Josephs O, Frith CD, Friston KJ, Frackowiak RS, Turner R (1997) Characterizing the relationship between BOLD contrast and regional cerebral blood flow measurements by varying the stimulus presentation rate. NeuroImage 6:270-278.

Sato C, Nemoto M, Tamura M (2002) Reassessment of activity-related optical signals in somatosensory cortex by an algorithm with wavelengthdependent path length. Jpn J Physiol 52:301-312.

Sheth S, Nemoto M, Guiou M, Walker M, Pouratian N, Toga AW (2003) Evaluation of coupling between optical intrinsic signals and neuronal activity in rat somatosensory cortex. NeuroImage 19:884-894. 
Sheth S, Nemoto M, Guiou M, Walker M, Pouratian N, Hageman N, Toga AW (2004) Columnar specificity of microvascular oxygenation and volume responses: implications for functional brain mapping. J Neurosci 24:634-641.

Shoham D, Grinvald A (2001) The cortical representation of the hand in macaque and human area S-I: high resolution optical imaging. J Neurosci 21:6820-6835.

Siegel AM, Culver JP, Mandeville JB, Boas DA (2003) Temporal comparison of functional brain imaging with diffuse optical tomography and fMRI during rat forepaw stimulation. Phys Med Biol 48:1391-1403.

Simons DJ, Carvell GE (1989) Thalamocortical response transformation in the rat vibrissa/barrel system. J Neurophysiol 61:311-330.
Thompson JK, Peterson MR, Freeman RD (2003) Single-neuron activity and tissue oxygenation in the cerebral cortex. Science 299:1070-1072.

Vanzetta I, Grinvald A (1999) Increased cortical oxidative metabolism due to sensory stimulation: implications for functional brain imaging. Science 286:1555-1558.

Villringer A, Them A, Lindauer U, Einhaupl K, Dirnagl U (1994) Capillary perfusion of the rat brain cortex. An in vivo confocal microscopy study. Circ Res 75:55-62.

Woolsey TA, Rovainen CM, Cox SB, Henegar MH, Liang GE, Liu D, Moskalenko YE, Sui J, Wei L (1996) Neuronal units linked to microvascular modules in cerebral cortex: response elements for imaging the brain. Cereb Cortex 6:647-660. 